\title{
SUZ12 Depletion Suppresses the Proliferation of Gastric Cancer Cells
}

\author{
Yingjun Cui ${ }^{a}$ Jie Chen ${ }^{b}$ Zhixian $\mathrm{He}^{c} \quad$ Yongtao Xiao $^{\mathrm{b}}$ \\ aDepartment of Surgery, Yantaishan Hospital, Yantai, China; ${ }^{b}$ Department of Pediatric Surgery, Xin Hua \\ Hospital, School of Medicine, Shanghai Jiao Tong University, Shanghai, China; 'Department of General \\ Surgery, School of Medicine, Affiliated Hospital of Nantong University, Nantong, China
}

\section{Key Words}

Gastric cancer $\bullet$ SUZ12 Proliferation • Epigenetic • MicroRNA

\begin{abstract}
Background/Aims: SUZ12 and EZH2 are two main components of polycomb repressive complex 2 (PRC2) that is known to be of great importance in tumorigenesis. EZH2 has been reported to play a vital role in pathogenesis of human cancer. However, whether SUZ12 has equivalent roles in tumorigenesis has not been demonstrated. Here, we investigated a possible role of SUZ12 for the proliferation of gastric cancer cells. Methods: Western-blot analysis was used to detected the levels of SUZ12, H3K27me3, EZH2 and p27 in ten gastric cell lines. SUZ12 was depleted by RNA interference. Cell cycle was detected by flow cytometry. Luciferase assays was to analyze whether miR-200b directly regulate SUZ12. Results: We found that SUZ12 depletion mediated by RNA interference (RNAi) led to a reduction of gastric cell numbers and arrested the cell cycle at G1/S point. As an important G1/S phase inhibitory gene, p27 is re-induced to some extent by SUZ12 knockdown. Furthermore, we demonstrated that SUZ12 was directly downregulated by miR-200b. Conclusion: We provide evidence suggesting that SUZ12 may be a potential therapeutic target for gastric cancer.
\end{abstract}

Copyright (C) 2013 S. Karger AG, Basel

\section{Introduction}

SUZ12 is an essential component of Polycomb Repressive Complex2 (PRC2), which modifies transcription by affecting histone and DNA methylation [1]. SUZ12 has been involved in both histone methyltransferase activity and the silencing function of the EED-EZH2 complex [2]. SUZ12 is hard to detect in normal tissues but amplified and overexpressed in several human cancers, such as oarian cancer, mantle cell lymphoma or breast cancer [3-5]. 
There is increasing evidence showes that SUZ12 plays an important role in carcinogenesis by acting as an oncogene, such as stimulates cell proliferation, blocks apoptosis, and promotes cell invasion and metastasis [3-7].

Gastric cancer is the fourth most common cancer worldwide with 930,000 cases diagnosed in 2002. It is a disease with a high death rate $(\sim 800,000$ per year $)$ making it the second most common cause of cancer death worldwide after lung cancer [8,9]. As another subunit, EZH2 was demonstrated to play a vital role in pathogenesis of human gastric cancer [10-12]. However, investigations of the SUZ12 status and function in gastric cancer cells are still unknown. In the present work, we will address this issue by investigating the contribution of SUZ12 to the growth of gastric cancer cell lines.

MicroRNAs (miRNAs) are approximately 21-23 nucleotides long, non-coding RNA, which regulate gene expression at the post-transcriptional level. The members of miR-200 family have been demonstrated to decrease in gastric cancer. [13] In addition, Iliopoulos et al. reported that the miR-200b directly down-regulated expression of SUZ12 in breast cancer stem cells. [4] Here, we are aslo going to address the question that whether miR-200b affects the proliferation of gastric cancer cells through targeting the SUZ12.

\section{Materials and Methods}

\section{Cell culture and transfections}

The MKN1, MKN45, MKN74, AZ521, KATO-III, IM95, IM95M, GTL16, NUGC3 and NUGC4 gastric cancer cells were bought from American Type Culture Collection (ATCC) and cultured in the RPMI media 1640 with $10 \%$ fetal bovine serum (FBS) at 37 degrees, $5 \% \mathrm{CO}_{2}$. The SUZ12 siRNA vector was transfected into gastric cells by lipofectamine 2000 (Invitrogen). MKN45, MKN74, AZ521 cells were plated in 6-well plates a day before grown to $60 \%$ confluence. Cells were transfected with $50 \mathrm{pM}$ of silencer negative control miRNA (Ambion) or miR-200b precursors (Ambion) with lipofectamine RNAiMAX (Invitrogen).

\section{Western-blot analysis}

20 ug protein each well was performed on $4-12 \%$ SDS-polyacrylamide gels and transferred onto nitrocellulose (NC) membranes with dry blotting system (Invitrogen). After blocking in 5\% nonfat dry milk, the membranes were incubated with the primary antibodies in blocking buffer $(1 \times \mathrm{TBST}, 3 \%$ nonfat dry milk, $0.2 \%$ Tween 20 ) for overnight at $4{ }^{\circ} \mathrm{C}$. The membranes were washed with $1 \times \mathrm{TBST}$ and then incubated with secondary antibodies. After final washes with $1 \times$ PBS, $0.2 \%$ Tween 20 , the signals were detected using ECL chemiluminescence reagents (Pierce). Antibodies of SUZ12, EZH2, H3K27me3, p27 and GAPDH (Cell signal technology) were used in this study.

\section{Proliferative and Flow cytometric analysis}

Cells were seeded in 96-well plates with 30-50\% confluence initially. The proliferation of gastric cells was analyzed using the Cell Counting Kit-8 (Dojindo) after 24, 48 or 72 hours. The absorbance value of each well was determined at $450 \mathrm{~nm}$ by a microplate reader (Molecular Devices, Beckman). Cells were fixed with ice-cold $70 \%$ ethanol, labeled with PI, and cell cycle was detected by flow cytometry as described before [14].

\section{Luciferase assays}

MKN45, MKN74, AZ521 cells were transfected with firefly luciferase reporter (pRL4.51) constructs containing the 3'UTR of SUZ12, (Including a wild type or mutant type containing two substitutions (CAGTATTA to CACTACTA) ), together with $100 \mathrm{nM}$ miRNA negative control, miR-200b by using Lipofectamine 2000. Cell extracts were prepared 48 hours after transfection. The Luciferase activity was measured with using the Dual Luciferase Reporter Assay System (Promega).

\section{Chromatin immunoprecipitation assay}

The chromatin in MKN45 cells were cross-linked with formaldehyde and sheared to fragment DNA. The samples were then immunoprecipitated with agarose-conjugated H3K27me3 (cell signal technology) 
Fig. 1. SUZ12 expression in gastric cancer cell lines. Immunoblot analysis of SUZ12 protein expression was performed on ten gastric cancer cell lines. It indicated SUZ12 is expressed abundantly in these cell lines.

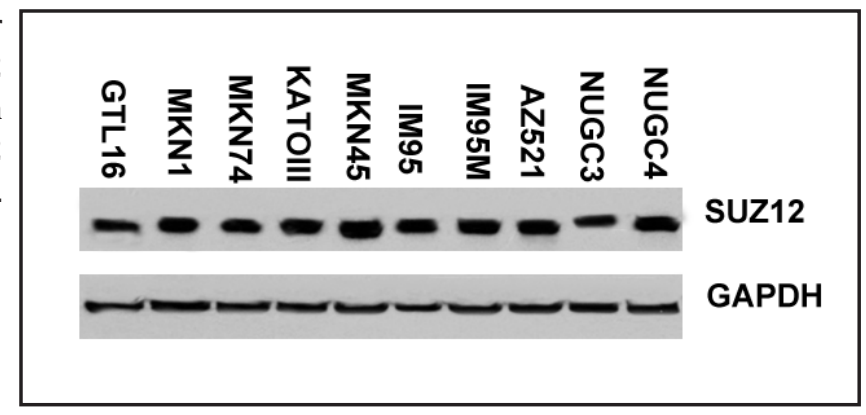

antibody or IgG control at $4{ }^{\circ} \mathrm{C}$ overnight. The following immunoprecipitation was performed according to the manufacturer's protocol (Upstate Biotechnologies, Charlottesville, VA). Brifely, crosslinks were removed at $65^{\circ} \mathrm{C}$ for $6 \mathrm{~h}$, and immunoprecipitated DNA was purified by phenol/chloroform extraction and ethanol precipitation. In the immunoprecipitated samples, the p27 promoter was determined by PCR.

\section{Statistical Analysis}

All data are reported as mean \pm S.E. When comparisons were made between two different groups, statistical significance was determined by the Student's t-test using the SPSS19 software program. P values from a two-tailed test were $<0.05$ were considered statistically significant.

\section{Results}

The growth of gastric cancer cells was inhibited significantly by SUZ12 repression

In order to investigate the role of SUZ12 in the growth of gastric cancer cells, we initially analyzed the expression of SUZ12 in a panel of ten tumor-derived gastric cancer cell lines by western-blot. We found that all tested cell lines expressed considerable SUZ12 protein (Fig. 1). Subsequently, we generated a siRNA that targeting the region of the SUZ12 transcript and transfected into these gastric cancer cell lines. After 72 hours, the expression of SUZ12 and its targeted H3K27me3 protein were blocked efficiently in all these tested cell lines with western-blot analysis (Fig. 2). We next determined the effect of SUZ12 depletion on the proliferation of these ten gastric cancer cell lines. RNAi-mediated suppression of SUZ12 expression led to a significant reduction of cell numbers, which was clearly visible 48-72 hours following transfection of SUZ12 siRNAs.

SUZ12 repression led to G1 arrest and re-induced expression of p27 in gastric cancer cells Cellular proliferation is regulated primarily by the regulation of cell cycle, which consists of four distinct sequential phases, quiescent (G0), prereplicative (G1), DNA synthesis (S), and mitosis (M) states. Here, we performed cell cycle analysis via propidium iodide (PI) staining and flow cytometry. It revealed that a significant increase in G1 population and a concomitant decrease in S phase population after SUZ12 knockdown (Fig. 3B). These results indicated that SUZ12 repression caused cell cycle arrested at the G1/S point and therefore might inhibited growth of gastric cancer cells. p27 is a cyclin dependent kinase whose major target is the cyclinE/CDK2 complex that governs cell cycle transition from late G1 to $S$ phase [15]. Here, we found that RNAi-mediated silencing SUZ12 was associated with a substantial re-increase of p27 expression at protein level (Fig. 3, 4). Futhermore, chromatin immunoprecipitation assay showed that depletion of SUZ12 reduced the methylation of p27 promoter significantly when compared to the controls (Fig. 5). Therefore, it is suggested that over-expressed SUZ12 in gastric cancer cells may contribute to the reduction of p27 expression by hymethylating its promoter.

MiR-200b directly regulated SUZ12 expression in gastric cells

The miR-200 family was found to be down-regulated in gastric carcinoma cell lines, especially those infected with recombinant Epstein-Barr virus (EBV). [13] it reported that 


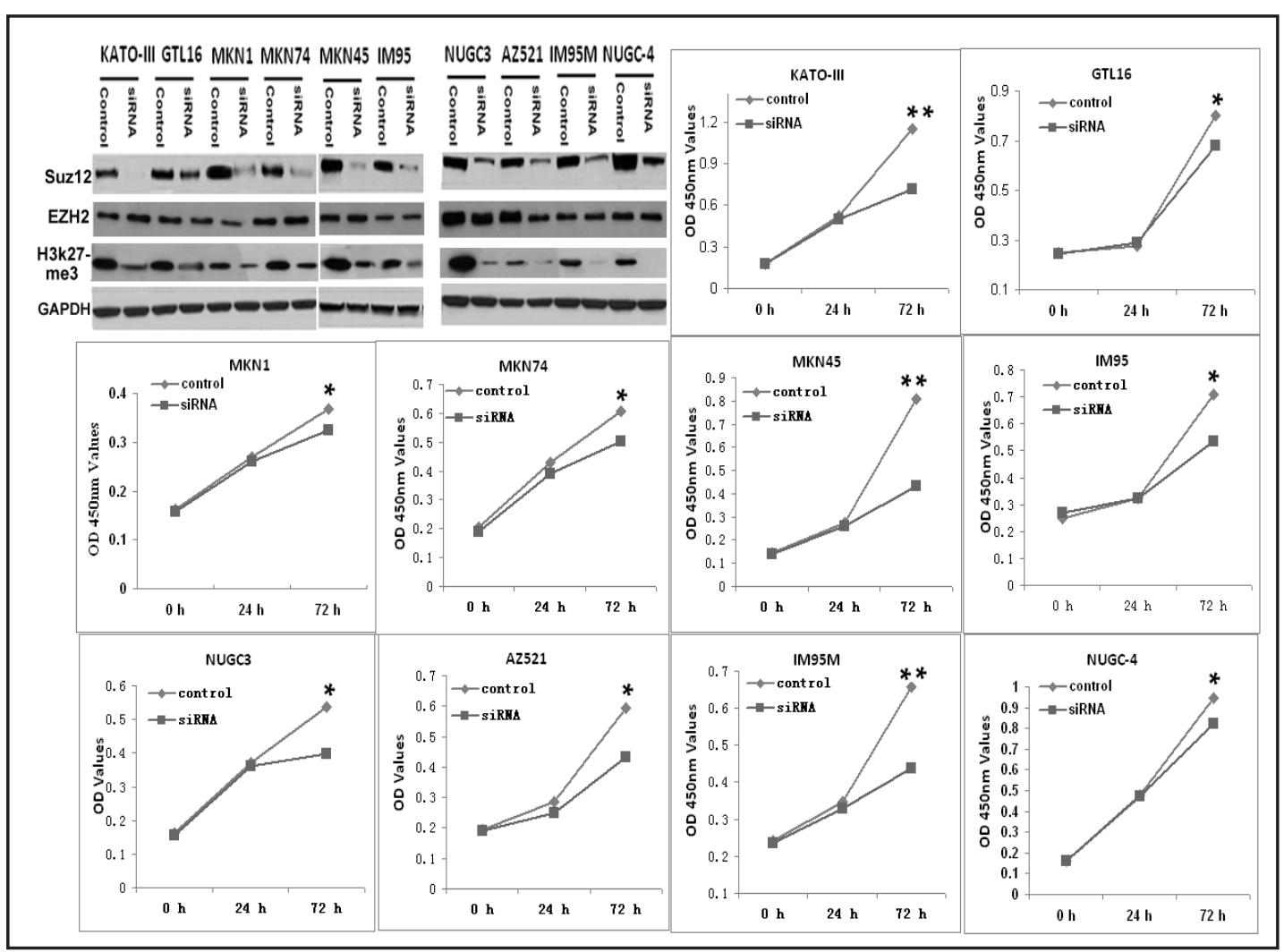

Fig. 2. SUZ12 repression led to growth inhibition of gastric cancer cell lines. Proliferative assay and western-blot were carried out following transient transfection with control siRNA or SUZ12-targeting siRNAs. It showed that the expression of SUZ12 protein was inhibited significantly after transfected with SUZ12 siRNA. Graphs represent relative cell proliferation at the indicated time points after siRNA transfection. The cells number was reduced significantly after SUZ12 depletion. Asterisks equal $p<0.05$, Double Asterisks equal $\mathrm{p}<0.01$.

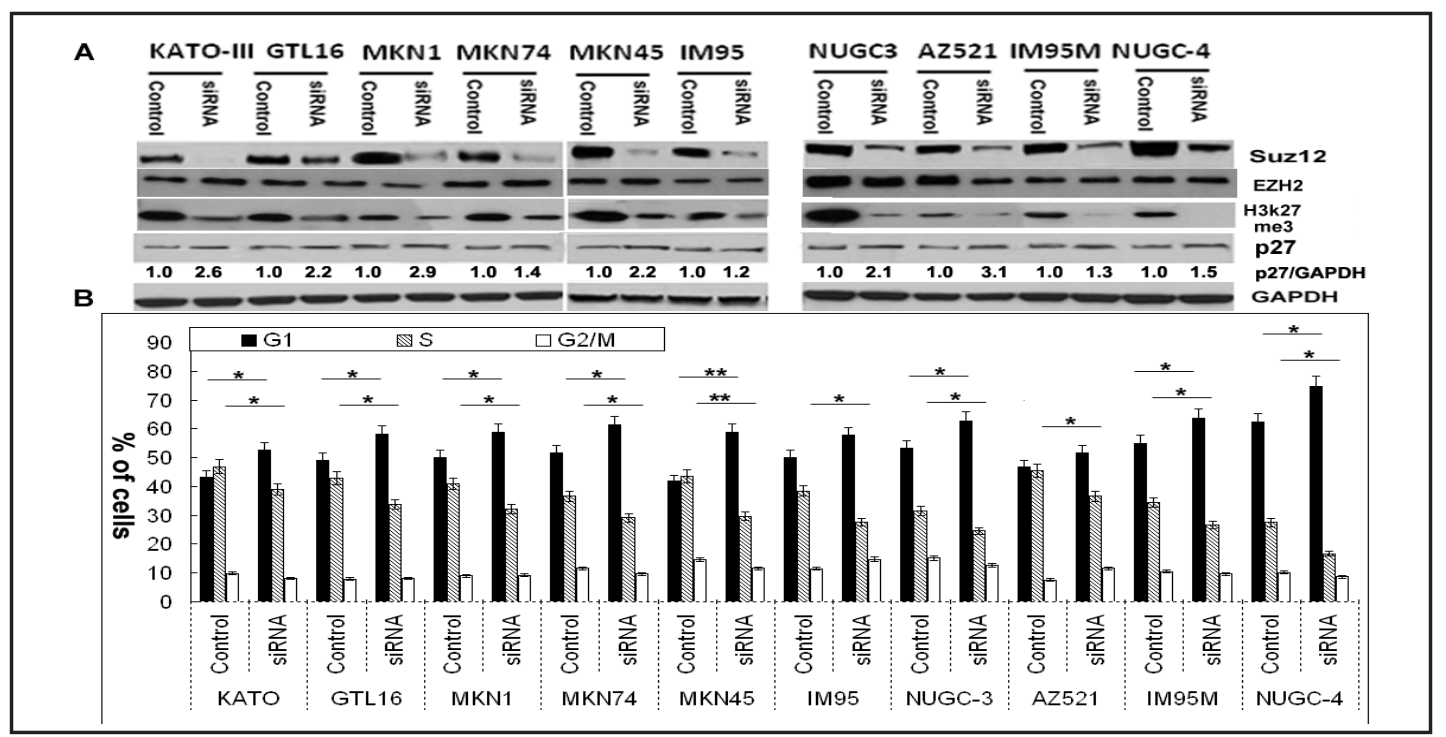

Fig. 3. SUZ12 repression led to cell cycle arrest at G1/S boundary. Immunoblot analyses showed that efficient downregulation of SUZ12 expression and re-induced expression of p27 by SUZ12 RNAi (A). Cell cycle analyses by FACS. Percentages of cells in the G1, S, or G2/M phases of the cell cycle were indicated. Asterisks equal $\mathrm{p}<0.05$, Double Asterisks equal $\mathrm{p}<0.01$. 


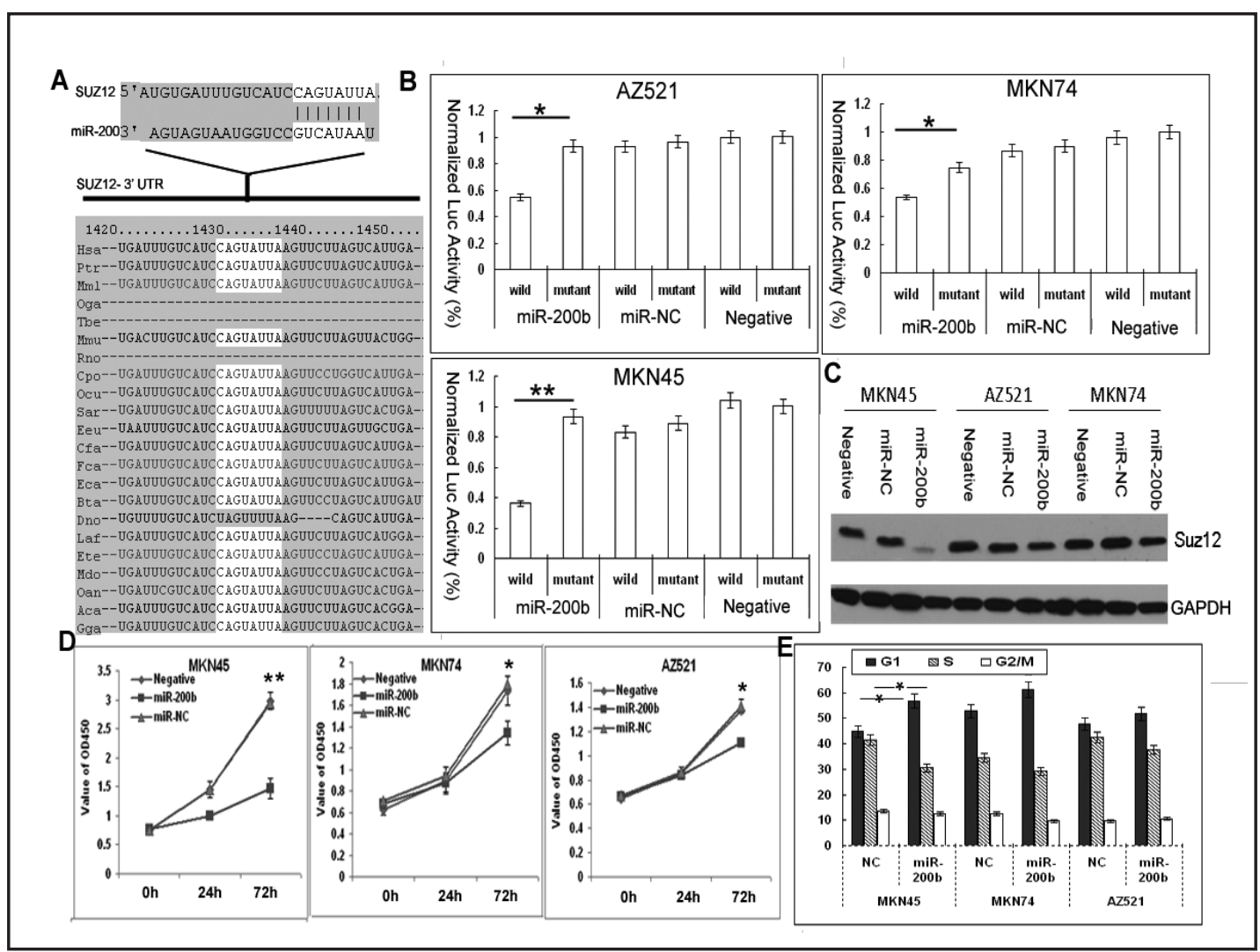

Fig. 4. SUZ12 is one of direct targets of miR-200b in gastric cancer cells. Predicted miR-200b binding sites in the 3'UTRs of SUZ12 with sequence complementarity and phylogenic conservation of 8 nt seed sequences. The miRNA database TargetScan version 6.2 (http://www.targetscan.org) was used to identify potential miRNA targets for miR-200b and to compare the miR-20b seed sequences of SUZ12 3'UTRs among different species. SUZ12 3' UTR report constructs, containing a wild type and a mutant were co-transfected into MKN45, MKN74 and AZ521 cells which were transfected by miR-control or miR-200b for 48 hours. Relative repression of firefly luciferase expression was standardized to a transfection control. The activity of luciferase was inhibited significantly by miR-200b introduction in MKN45, MKN74 and AZ521 cells. Western-blot analysis indicated that miR-200b repressed the expression of SUZ12 in these cell lines (A-C). The administration of miR-200b led to a significant reduction of cell number and caused cell cycle arresting at G1/S point (D, E).

Fig. 5. The depletion of SUZ12 re-expressed p27. MKN45 cells were transfected with a control vector or the SUZ12 siRNA. At $48 \mathrm{~h}$ posttransfection, genomic chromatin fragments were immunoprecipitated with $\mathrm{H} 3 \mathrm{~K} 27 \mathrm{me} 3$ at the p27 promoter. PCR analysis on input chromatin (first two lanes) confirmed that equal chromatin amounts were used for chromatin immunoprecipitation.

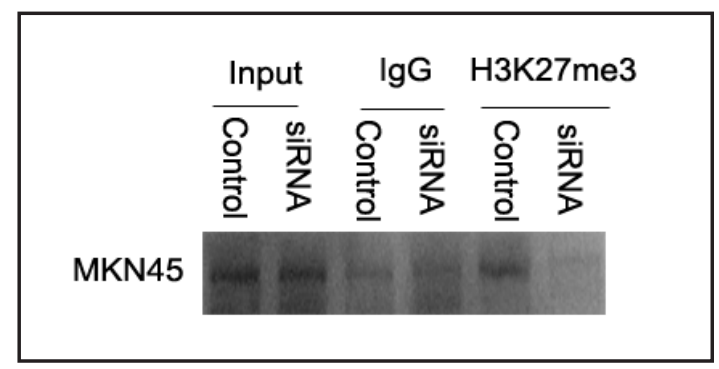

miR-200b could directly target SUZ12 in breast cancer stem cells. [4] Here, we found that miR-200b inhibited the expression of SUZ12 in MKN45, MKN74 and AZ521 through directly binding to its 3' UTR region. As shown in the Figure 4, it is perfectly complementary between 
miR-200b seed sequence and SUZ12 3'UTR sequence that is highly conserved among species. Dual-luciferase assay was a standard experiment, which was usually used to confirm the direct targets of miRNAs. We found that the activity of luciferase was inhibited significantly by miR-200b in MKN45, MKN74 and AZ521 cells. Moreover, at protein level, the expression of SUZ12 was suppressed by miR-200b introduction in these cells. Interestingly, the miR-200b mediated-inhibitory effect on the SUZ12 is more significant in MK45 than in another two cell lines (Fig. 4A-C). The MKN45, MKN74 and AZ521 cell lines that transfected with miR-200b mimics led to cell cycle arrest at G1/S and a significant reduction in cell numbers (Fig. 4D, E). Summarily, these results suggested miR-200b could down-regulated SUZ12 directly through binding 3' UTR of SUZ12.

\section{Discussion}

In our present study, we showed that ten gastric cancer cell lines expressed amounts of SUZ12 protein. Next, we knocked down the SUZ12 efficiently in the tested cells with SUZ12 siRNA. After SUZ12 depletion, the gastric cancer cell number decreased in short term and cell cycle arrested at the G1/S boundary. However, the exact molecular mechanisms how SUZ12 depletion blocks the gastric cancer cells proliferation of are unclear. EZH2, another crucial component of the PRC2 transcriptional repressor complex, could repress the anitiproliferative genes in several types of tumors [16-20]. A previous study reported that EZH2 led to repression of the growth-inhibitory p27 cell cycle regulator gene in pancreatic cancer cells [21]. Whether SUZ12 has equivalent roles in p27 suppression is still unknown. In this study, we found that SUZ12 knockdown resulted in a re-expression of p27 in several tested gastric cancer cell lines in vitro. In addition, we examined the DNA methylation status of p27 in this process of EZH2 knockdown. The methylated p27 promoter was diminished significantly after SUZ12 RNAi treatment, suggesting that SUZ12 is closely related to the methylation of the p27 promoter and its maintenance. Since p27 acts at the G1/S transition, it indicated that the SUZ12 depletion led to cell cycle arrest at G1/S boundary might through re-induction of p27 expression.

The miR-200 family mainly targeted the ZEB1 and ZEB2 transcriptional repressors of $\mathrm{CDH} 1$ and severs as a potential suppressor of epithelial-mesenchymal transition or transformation (EMT) [22, 23]. In a recent report, Iliopoulos et al. demonstrated that miR$200 \mathrm{~b}$ also targeted the SUZ12 through a direct interaction with a perfectly homologous and highly conserved region of the SUZ12 3'UTR. It was indicated that decreases in miR-200b lead to increased SUZ12 expression, increased binding of SUZ12 to the CDH1 promoter, and increased H3-K27 trimethylation and polycomb-mediated repression of E-cadherin expression. Here, we demonstrated that miR-200b could inhibit the expression of SUZ12 and suppress cell growth in MKN45, MKN74 and AZ521 cells. Surprisingly, in MKN45 cells, the function of miR-200b was more significant in terms of SUZ12 suppression and cell growth inhibition. It indicated that miR-200b might repress expression of SUZ12 in a celltype-dependent way. MKN45 is a stem-like cell line, which was established from the poorly differentiated adenocarcinoma of the stomach. MKN74 and AZ521 were differentiated gastric cancer cells. It could be hypothesized that miR-200b mightily control the expression of SUZ12 just in undifferentiated cells, such as cancer stem cells that reported by Iliopoulos et al. [4].

\section{References}

1 Pasini D, Bracken AP, Jensen MR, Lazzerini Denchi E, Helin K: Suz12 is essential for mouse development and for ezh2 histone methyltransferase activity. EMBO J 2004;23:4061-4071.

2 Cao R, Zhang Y: Suz12 is required for both the histone methyltransferase activity and the silencing function of the eed-ezh2 complex. Mol Cell 2004;15:57-67. 
Cui/Chen/He/Xiao: SUZ12 and Gastric Cell Proliferation

3 Li H, Cai Q, Wu H, Vathipadiekal V, Dobbin ZC, Li T, Hua X, Landen CN, Birrer MJ, Sanchez-Beato M, Zhang R: Suz12 promotes human epithelial ovarian cancer by suppressing apoptosis via silencing hrk. Mol Cancer Res 2012;10:1462-1472.

4 Iliopoulos D, Lindahl-Allen M, Polytarchou C, Hirsch HA, Tsichlis PN, Struhl K: Loss of mir-200 inhibition of suz12 leads to polycomb-mediated repression required for the formation and maintenance of cancer stem cells. Mol Cell 2010;39:761-772.

5 Martin-Perez D, Sanchez E, Maestre L, Suela J, Vargiu P, Di Lisio L, Martinez N, Alves J, Piris MA, SanchezBeato M: Deregulated expression of the polycomb-group protein suz12 target genes characterizes mantle cell lymphoma. Am J Pathol 2010;177:930-942.

6 Li H, Ma X, Wang J, Koontz J, Nucci M, Sklar J: Effects of rearrangement and allelic exclusion of jjaz1/suz12 on cell proliferation and survival. Proc Natl Acad Sci U S A 2007;104:20001-20006.

7 Hinz S, Kempkensteffen C, Christoph F, Krause H, Schrader M, Schostak M, Miller K, Weikert S: Expression parameters of the polycomb group proteins bmi1, suz12, ring1 and cbx7 in urothelial carcinoma of the bladder and their prognostic relevance. Tumour Biol 2008;29:323-329.

-8 Jemal A, Bray F, Center MM, Ferlay J, Ward E, Forman D: Global cancer statistics. CA Cancer J Clin 2011;61:69-90.

-9 Parkin DM: Epidemiology of cancer: Global patterns and trends. Toxicol Lett1998;102-103:227-234.

10 He LJ, Cai MY, Xu GL, Li JJ, Weng ZJ, Xu DZ, Luo GY, Zhu SL, Xie D: Prognostic significance of overexpression of ezh2 and h3k27me3 proteins in gastric cancer. Asian Pac J Cancer Prev 2012;13:3173-3178.

11 Carvalho J, van Grieken NC, Pereira PM, Sousa S, Tijssen M, Buffart TE, Diosdado B, Grabsch H, Santos MA, Meijer G, Seruca R, Carvalho B, Oliveira C: Lack of microrna-101 causes e-cadherin functional deregulation through ezh2 up-regulation in intestinal gastric cancer. J Pathol 2012;228:31-44.

12 Mattioli E, Vogiatzi P, Sun A, Abbadessa G, Angeloni G, D'Ugo D, Trani D, Gaughan JP, Vecchio FM, Cevenini G, Persiani R, Giordano A, Claudio PP: Immunohistochemical analysis of prb2/p130, vegf, ezh2, p53, p16(ink4a), p27(kip1), p21(waf1), ki-67 expression patterns in gastric cancer. J Cell Physiol 2007;210:183191.

13 Shinozaki A, Sakatani T, Ushiku T, Hino R, Isogai M, Ishikawa S, Uozaki H, Takada K, Fukayama M: Downregulation of microrna-200 in ebv-associated gastric carcinoma. Cancer Res 2010;70:4719-4727.

-14 Iredale JP, Benyon RC, Pickering J, McCullen M, Northrop M, Pawley S, Hovell C, Arthur MJ: Mechanisms of spontaneous resolution of rat liver fibrosis. Hepatic stellate cell apoptosis and reduced hepatic expression of metalloproteinase inhibitors. J Clin Invest 1998;102:538-549.

15 Philipp-Staheli J, Payne SR, Kemp CJ: P27(kip1): Regulation and function of a haploinsufficient tumor suppressor and its misregulation in cancer. Exp Cell Res 2001;264:148-168.

-16 Zhang H, Zhao M, Lv Z, Zhang X, Qin X, Wang H, Wang S, Su J, Lv X, Liu H, Du W, Zhou W, Chen X, Fei K: Mir138 inhibits tumor growth through repression of ezh2 in non-small cell lung cancer. Cell Physiol Biochem 2013;31:56-65.

17 Kikuchi J, Takashina T, Kinoshita I, Kikuchi E, Shimizu Y, Sakakibara-Konishi J, Oizumi S, Marquez VE, Nishimura M, Dosaka-Akita H: Epigenetic therapy with 3-deazaneplanocin a, an inhibitor of the histone methyltransferase ezh2, inhibits growth of non-small cell lung cancer cells. Lung Cancer 2012;78:138-143.

18 Xia H, Yu CH, Zhang Y, Yu J, Li J, Zhang W, Zhang B, Li Y, Guo N: Ezh2 silencing with rnai enhances irradiation-induced inhibition of human lung cancer growth in vitro and in vivo. Oncol Lett 2012;4:135140.

19 Chen Y, Xie D, Yin Li W, Man Cheung C, Yao H, Chan CY, Xu FP, Liu YH, Sung JJ, Kung HF: Rnai targeting ezh2 inhibits tumor growth and liver metastasis of pancreatic cancer in vivo. Cancer Lett 2010;297:109-116.

20 Karanikolas BD, Figueiredo ML, Wu L: Comprehensive evaluation of the role of ezh2 in the growth, invasion, and aggression of a panel of prostate cancer cell lines. Prostate 2010;70:675-688.

21 Ougolkov AV, Bilim VN, Billadeau DD: Regulation of pancreatic tumor cell proliferation and chemoresistance by the histone methyltransferase enhancer of zeste homologue 2 . Clin Cancer Res 2008;14:6790-6796.

22 Korpal M, Lee ES, Hu G, Kang Y: The mir-200 family inhibits epithelial-mesenchymal transition and cancer cell migration by direct targeting of e-cadherin transcriptional repressors zeb1 and zeb2. J Biol Chem 2008;283:14910-14914.

23 Park SM, Gaur AB, Lengyel E, Peter ME: The mir-200 family determines the epithelial phenotype of cancer cells by targeting the e-cadherin repressors zeb1 and zeb2. Genes Dev 2008;22:894-907. 